\title{
UNA CLASIFICACION DE LOS ADJETIVOS EN GRIEGO
}

\begin{abstract}
The purpose of this paper is to show that four subclasses of adjectives can be set up in Ancient Greek. To such an aim a number of morphosyntactic tests is used in order to find out the features shared by each group of adjectives as well as the properties keeping each subclass apart. In the light of the applied tests, four groups are established; they can be called, according to their semantic features: determiners, identifiers, quantifiers and qualifiers. It goes without saying that it is to be counted with the existence of historical changes as to the actual subclassification of each item.
\end{abstract}

§1. Sobre los criterios para clasificar las partes de la oración. Los criterios que permiten clasificar las partes de la oración en una lengua son fonológicos, morfológicos, sintácticos y semánticos. Las definiciones de las partes de la oración que se encuentran en las gramáticas tradicionales usan indiscriminadamente unos $\mathrm{u}$ otros criterios, aunque los de tipo semántico son los predominantes. En efecto, en la gramática griega tradicional, unas partes de la oración son identificadas con ayuda de criterios semánticos (como en ciertas definiciones del nombre y del verbo), y otras partes de la oración son definidas de acuerdo con sus características sintácticas (adjetivo como modificador del nombre; adverbio como modificador de un verbo, un adjetivo $u$ otro adverbio). No faltan tampoco otras definiciones basadas en criterios fonológicos o en el orden de palabras (como en las preposiciones y el artículo) 1.

Frente a este procedimiento heterogéneo de la gramática tradicional, se ha señalado con frecuencia la necesidad metodológica de identificar

1 En realidad, es probable - aunque, de hecho, es sólo una hipótesis difícilmente verificable - que exista una correspondencia exacta entre los resultados a los que conduce la aplicación de cada uno de los criterios que se usan para clasificar las partes de la oración en una lengua dada. Si existen discrepancias entre las conclusiones a las que conducen los diferentes criterios, se puede presumir que alguno de ellos no ha sido aplicado de modo correcto. 
las partes de la oración mediante criterios formales y sintácticos o distribucionales. Las causas que hacen necesario tal proceder son bien conocidas, y no hace falta insistir aquí sobre los peligros inherentes a un enfoque puramente semántico. No obstante, mediante la aplicación sistemática de criterios formales y sintácticos únicamente se logran identificar clases a las que hay que designar necesariamente de un modo convencional (clase A, B, C, etc.). Sólo la aplicación de criterios semánticos en un momento posterior de la investigación permite asignar una definición significativa a las clases previamente establecidas. De esta suerte, se puede denominar, por ejemplo, la clase A como la de los verbos, la $\mathrm{B}$ como la de los nombres, la $\mathrm{C}$ como la de los adjetivos, etc. Es decir, hay que distinguir, teóricamente al menos, dos momentos diferentes en la investigación: por un lado, la observación de que una serie de palabras es susceptible de compartir un conjunto de formas y ejercer ciertas funciones sintácticas; por otro lado, la definición de los grupos previamente establecidos ${ }^{2}$.

El propósito de las páginas que siguen es mostrar que con ayuda de estos mismos criterios, aplicados en el mismo orden en la investigación -es decir, primero los formales y sintácticos, y posteriormente los semánticos, si ello es posible-, se puede lograr una clasificación más precisa de las partes de la oración, y en concreto de los adjetivos. En éstos, en efecto, existen diferencias -morfosintácticas y semánticas- suficientes para distinguir los siguientes grupos: calificativos, cuantificadores, determinativos y un grupo algo más heterogéneo, al que aquí denominaremos identificadores ${ }^{3}$. La denominación que damos a cada una de estas clases descansa sobre una caracterización semántica; pero, como trataremos de hacer ver, la identificación de estos grupos está basada sobre todo en ciertas propiedades morfosintácticas, peculiares de cada clase de adjetivos. La definición semántica sólo se puede obtener tras la aplicación de los demás criterios; sin embargo, para

2 Para la distinción entre definición de las partes de la oración (nivel correspondiente al objeto de la lingüística general) y clasificación de palabras en partes de la oración (nivel correspondiente al objeto del estudio de cada lengua particular), cf. E. Coseriu, "Sobre las categorías verbales ('partes de la oración')", Gramática, semántica, universales, Madrid 1978, p. 50 ss.

3 En realidad, esta clasificación es parcialmente usada por Schwyzer-Debrunner, p. 173 ss.; L. Bergson, Zur Stellung des Adjektivs in der älteren griechischen Prosa, Upsala 1960, que, a su vez, la toma de J. Marouzeau, L'ordre des mots dans la phrase latine. I. Les groupes nominaux, París 1922; L'ordre des mots dans la phrase latine. Volume complémentaire, París 1953. Cf., en general, J. Lyons, Semantics 2, Cambridge 1977, p. 438 ss., así como la bibliografía que cita en p. 450 , n. 8. - Los adjetivos que denominamos «identificadores" no coinciden totalmente con los que cubre el término «determiners», cf. infra $\S 3$. 
conseguir una mayor claridad en la exposición y para evitar denominaciones convencionales como clase A, clase B, etc., de adjetivos, distinguiremos aquí desde el primer momento las clases diferentes de adjetivos mediante una definición semántica aproximada, que en el orden de la investigación se obtendría al final.

§ 2. Características del adjetivo en griego. El propósito de estas páginas es justificar la clasificación de los adjetivos expuesta más arriba. Quedan, pues, fuera de nuestra consideración algunas cuestiones más generales, que, sin embargo, es preciso examinar brevemente. De estas cuestiones, la más importante es saber qué propiedades comunes poseen los adjetivos en griego, y cuáles son las diferencias entre adietivo y nombre o verbo, sobre todo 4 .

Como es sabido, el adjetivo en griego y en las lenguas indoeuropeas en general se caracteriza por sus funciones sintácticas: el adjetivo es modificador opcional del nombre y ejerce las funciones sintácticas de predicado y atributo o expansión opcional de un nombre ${ }^{5}$. Más difícil, sin embargo, es hallar una caracterización semántica del adjetivo. La expresión de la cualidad, que es la noción semántica más frecuentemente invocada para la definición semántica de los adjetivos, no es específica

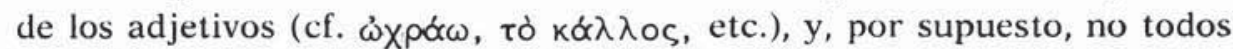

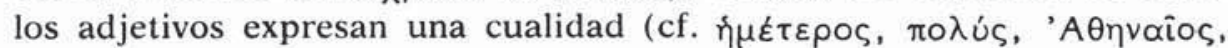
etcétera). Existe, desde luego, la posibilidad de considerar que los adjetivos que expresan una cualidad constituyen el "focal point" dentro del conjunto de los adjetivos, lo mismo que, al parecer, sucede con algunos nombres de colores, según la conocida tesis de B. Berlin y P. Kay ${ }^{6}$. En cualquier caso, es evidente que el adjetivo es una categoría verbal plenamente diferenciada en griego y caracterizada al menos por sus funciones sintácticas.

En cuanto a las diferencias que separan al adjetivo de otras partes de la oración, es preciso recordar en primer lugar que para los gramáticos de la Antigüedad el $\varepsilon \pi i \theta \varepsilon \tau o v$ es sólo una forma ( $\varepsilon i \delta \circ \varsigma$ ) del $\measuredangle v \circ \mu \alpha$ (Dion. Thr., p. 32 s. Uhlig). Existen realmente numerosas conexiones entre nombre y adjetivo, pero su separación como dos partes de la oración diferenciadas está justificada por diversas causas.

4 La relación de verbo y adjetivo es puesta de relieve en J. Lyons, op. cit., p. 447 ss., donde se encuentra la bibliografia pertinente.

5 Cf. Schwyzer-Debrunner, p. 11; Wackernagel, Vorlesungen, II, p. 51 ss.

6 Basic Color Terms, Berkeley - Los Ángeles 1969 (un resumen cómodo en español se encuentra en G. Leech, Semantics, trad. esp., Madrid 1977, p. 260 ss.). 
Aunque tanto el adjetivo como el nombre expresan las categorías gramaticales de género, número y caso, el adjetivo, sin embargo, puede expresar también la gradación, que sólo es esporádica en los nombres

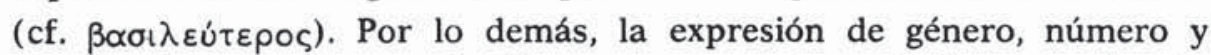
caso en los adjetivos es simplemente un producto de la concordancia, mientras que en los nombres la elección de una desinencia casual depende de la función sintáctica y del contenido semántico que se comunica, el género marca, al menos en parte, el sexo, y el número, igualmente, guarda cierta relación con el referente. Por tanto, aunque las categorías expresadas por el nombre y adjetivo son en parte las mismas, existen profundas diferencias.

En cuanto a las funciones sintácticas, existen también profundas diferencias entre nombre y adjetivo. Las funciones propias del adjetivo pueden ser también ejercidas por el nombre, con la única salvedad de que el nombre en función de atributo opcional recibe tradicionalmente la denominación de aposición ${ }^{7}$. En efecto, desde el punto de vista de la

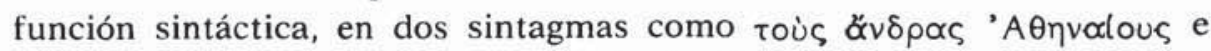
II. IX 479, $\Phi \theta i \eta v, \mu \eta \tau \varepsilon \rho \propto \mu \eta ́ \lambda \omega v$, tanto el adjetivo como la aposición son ampliaciones o expansiones opcionales de un nombre.

La diferencia sintáctica más importante entre adjetivo y nombre radica en el hecho de que el nombre puede ejercer todas las funciones sintácticas que expresa el adjetivo, pero éste no puede cumplir algunas funciones que son específicas del nombre. Así, mientras que cualquier nombre puede ser sujeto gramatical de la frase, ningún adjetivo en cuanto tal (es decir, no transformado en sustantivo) puede serlo. Esta circunstancia explica que la sustantivación de adjetivos sea un hecho usual, mientras que la adjetivación de nombres sea un fenómeno raro. En efecto, todos los sustantivos pueden cumplir las funciones sintácticas del adjetivo, y por eso no es preciso transformar nombres en adjetivos; pero los adjetivos no pueden cumplir, en cuanto tales adjetivos, algunas funciones sintácticas propias de los nombres, y por eso es preciso un mecanismo de transformación de adjetivos en nombres.

Tanto el nombre como el adjetivo pueden ser predicados de la oración. Sin embargo, esta aparente igualdad oculta ciertas diferencias. Entre los diversos tipos de predicados que podemos distinguir ${ }^{8}$, hay

7 Cf. la ordenación que sigue Schwyzer-Debrunner, p. 612 ss.

8 Cf., sobre todo, Ch. H. Kahn, The verb "bes in Ancient Greek, DordrechtBoston 1973, así como la recensión de C. J. Ruijgh, Lingua 48, 1979, pp. 43-83; J. Lyons, op. cit., p. 469 ss. 
que mencionar al menos un predicado atributivo y otro de identidad 9. Cada uno de ellos se puede ilustrar mediante las siguientes frases:

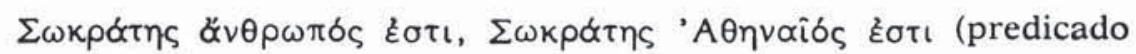
atributivo),

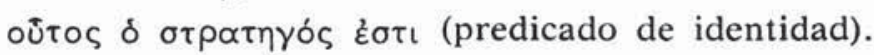

Mientras que el nombre puede ser predicado atributivo o de identidad, el adjetivo sólo puede ser predicado atributivo.

Hay que señalar que la distinción entre estos dos tipos de predicados no es sólo semántica, sino también sintáctica: en las oraciones con predicado de identidad no existe propiamente posibilidad de diferenciar sujeto y predicado, pues ambos son permutables; por el contrario, en las frases nominales con predicado atributivo no existe permutabilidad entre ambos. Bien es verdad que en griego el orden de palabras y el uso o ausencia del artículo son marcas formales que tienden a distinguir sujeto y predicado. No obstante, subsiste con frecuencia la ausencia de marcas formales que distingan sujeto y predicado. Así, el orden de palabras sugiere dos análisis diferentes en:

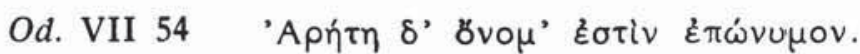

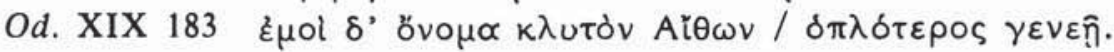

Tampoco el uso o la ausencia de artículo es un medio seguro que permita diferenciar sujeto y predicado en la poesía; e incluso en la prosa existe indistinción de ambos en frases como las siguientes:

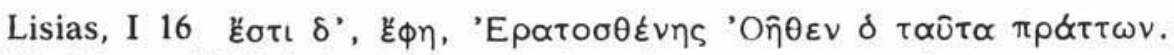

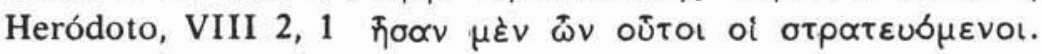

En conclusión, el adjetivo puede ser sólo predicado atributivo, mientras que el nombre puede ser predicado atributivo o de identidad ${ }^{10}$.

9 Los términos usados por J. Lyons son, respectivamente, «ascriptive" y «equativen.

10 La ocasional adjetivación de nombres guarda probablemente relación con la frecuencia de su uso como predicados atributivos. Así, se observa que los nombres que con mayor frecuencia son usados como predicados son los que expresan la edad, la profesión o la función social (cf. Wackernagel, Vorlesungen, II, p. 51 ss.; Schwyzer-Debrunner, p. 176; para el latín, A. Scherer, Handbuch der lateinischen Syntax, Heidelberg 1975, p. 191). Son precisamente estos nombres los que con mayor frecuencia han evolucionado a adjetivos o mantienen a lo largo de la historia del griego un estado fluctuante: nombres de agente en $-\tau \eta_{\rho},-\tau \omega \rho, \gamma \varepsilon \rho \omega \nu$, ciertos

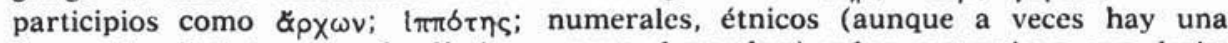
formación distinta para el adjetivo y para el nombre) y los sustantivos que designan un objeto que posee una cualidad específica (cf. en los nombres de colores rosa, violeta, etc.). 
Una vez caracterizado el adjetivo como parte independiente de la oración y señaladas las diferencias más importantes con respecto al nombre, debemos exponer ahora las propiedades morfosintácticas y semánticas que invitan a diferenciar las cuatro clases de adjetivos expuestas más arriba. Conviene advertir previamente, sin embargo, que no es nuestro propósito establecer una clasificación de los adjetivos que sea válida para cualquier lengua, sino sólo para el griego ".

§ 3. Clasificación de los adjetivos según criterios semánticos. La observación de ciertas características morfosintácticas y semánticas de los adjetivos en griego permite establecer una clasificación de los adjetivos en cuatro grupos: determinativos, calificativos, cuantificadores e identificadores. Antes de exponer las propiedades morfosintácticas de cada grupo, conviene presentar algunos ejemplos que ayuden a precisar qué adjetivos son incluidos en cada una de las cuatro clases semánticas. Es decir, trataremos de caracterizar el contenido semántico de cada clase de adjetivos, aun cuando, en rigor, ésta sería la etapa final de la investigación, sólo lograda después de la identificación de clases en función de las propiedades morfosintácticas. Sin embargo, la presentación previa de la clasificación semántica permitirá obtener mayor claridad en la exposición posterior.

Los determinativos expresan la pertenencia a un grupo o una pro-

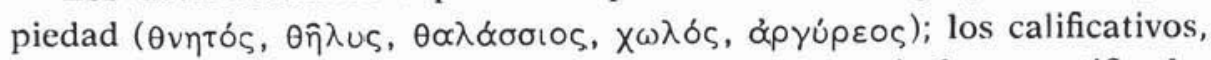

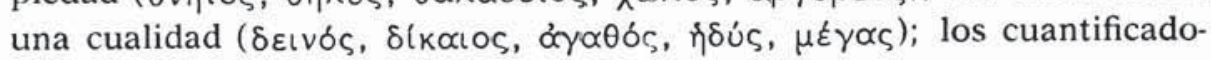

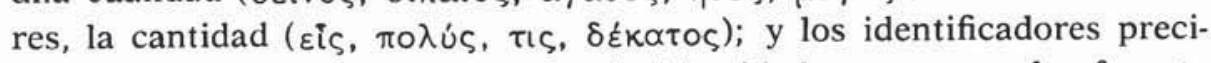
san de manera positiva o negativa la identidad que posee el referente

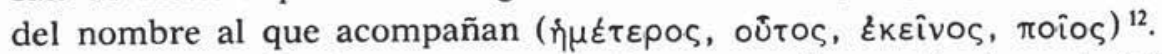

A los cuantificadores pertenecen los numerales, ordinales e indefinidos. El grupo de los identificadores está compuesto por los demostrativos, interrogativos y posesivos. Los calificativos expresan una cualidad $y$ en la medida en que implican un juicio personal son llamados por

I1 Para el latín, al menos, una clasificación semejante permite establecer la observación del orden de palabras habitual, cf. J. Marouzeau, op. cit., p. 1 ss., como indican las diferencias, por ejemplo, entre praetor urbanus y urbanus praetor. Para una clasificación ligeramente distinta en francés, cf. H. Weinrich, Sprache in Texten, trad. esp., Madrid 1981, p. 277 ss.

12 L. Bergson, op. cit. en nota 3, distingue determinativos, por un lado, y adjetivos de cantidad y de cualidad, por otro, siguiendo en parte a J. Marouzeau, I, p. 16 ss., que estudia separadamente los calificativos, los discriminativos (= determinativos), participios, posesivos y pronominales diversos. Una clasificación diferente en función del orden de palabras en griego propone J. Brunel, La construction de l'adjectif dans les groupes nominaux du grec, París 1964. 
Marouzeau ${ }^{13}$ "subjetivos". Los determinativos expresan la pertenencia a un grupo, la localización espacial o temporal o una propiedad física o material; son los llamados adjetivos "objetivos".

Si se toman, pues, como criterio principal de clasificación las propiedades morfosintácticas a las que nos referiremos en $\S \S 4$ y 5 , se puede presentar una serie de ejemplos característicos de los grupos semánticos diferenciados. Para la mejor comprensión de los ejemplos que siguen, hay que tener en cuenta ciertas precisiones de la mayor importancia:

a) Los adjetivos que componen la lista de ejemplos que sigue son clasificados en uno $\mathrm{u}$ otro grupo en función de las propiedades morfosintácticas expuestas en $\S \S 4$ y 5 ; no obstante, es también evidente la relación semántica existente entre los adjetivos que componen cada grupo.

b) La clasificación está basada en criterios internos propios del griego antiguo; por eso no hay que esperar que esta clasificación coincida con una posible clasificación semejante que se pueda establecer para otras lenguas indoeuropeas. Los miembros que integran cada grupo pueden ser en parte distintos en cada lengua, aun cuando la clasificación general sea válida para otras lenguas indoeuropeas.

c) Existen, sin duda, variaciones diacrónicas, cf. notas 14 y 15.

Teniendo en cuenta estas precisiones, se puede ilustrar cada uno de los grupos semánticos de adjetivos mediante la siguiente lista de ejemplos:

1. Cuantificadores: numerales, ordinales e indefinidos.

2. Identificadores: demostrativos, interrogativos y posesivos.

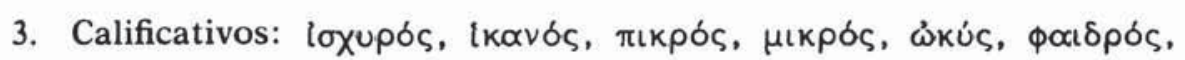

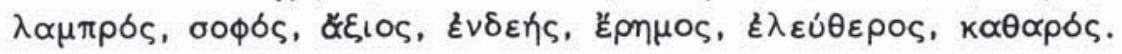

4. Determinativos:

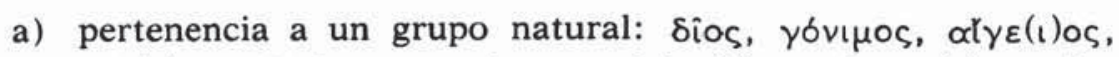

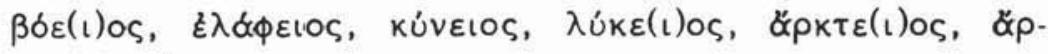

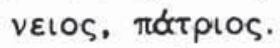

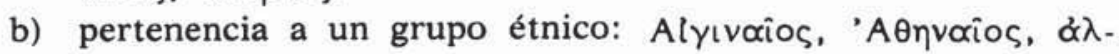
$\lambda \delta \phi u \lambda \circ \varsigma$.

13 Op. cit., p. 16 ss. La misma clasificación establece para el latín en función del orden de palabras J. M. Adams, IF 81, 1976, p. 88 ss. 
c) pertenencia a un grupo social o profesional: $\delta \circ \lambda^{\lambda} \iota \varsigma, \xi \xi$ -

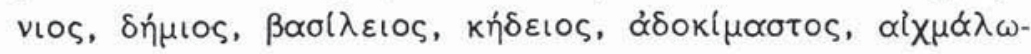

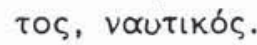

d) localización espacial: ápı

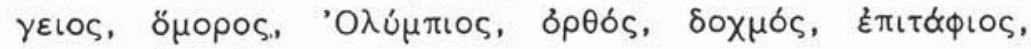

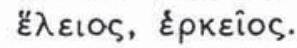



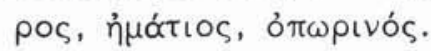

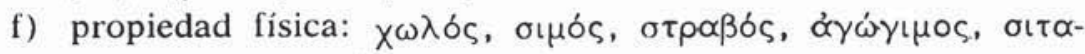
$\gamma \omega \gamma$

g) propiedad material: ¿ $\rho \gamma u ́ \rho \varepsilon \circ \varsigma, \sigma เ \delta \eta ́ p \varepsilon \circ \varsigma, \chi \rho u ́ \sigma \varepsilon \circ \varsigma, \chi \alpha \dot{\alpha} \kappa \varepsilon \circ \varsigma$, $\lambda i v \varepsilon \circ \varsigma, \pi \circ \rho \phi u ́ p \varepsilon \circ \varsigma, \alpha \lambda \mu u \rho \delta ́ \varsigma, \delta i \pi \tau u \chi \circ \varsigma, \delta 1 \pi \lambda \delta \circ \varsigma, \pi \lambda \alpha \tau u ́ \varsigma$.

La clasificación semántica no permite establecer una rígida distinción entre las diferentes clases, pues existen numerosos usos marginales. Así, la pertenencia puede ser ocasionalmente una cualidad (cf., por

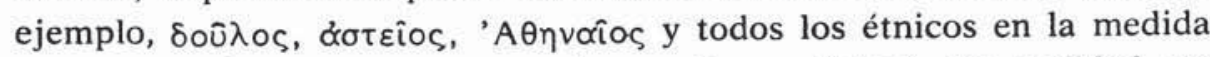
en que pueden expresar un modo peculiar o típico). En realidad, no existe diferencia entre una determinación que dura en el tiempo y una cualidad ${ }^{14}$. Los calificativos pueden expresar también una determina-

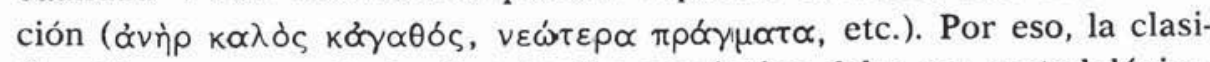
ficación que permite la descripción semántica debe ser metodológicamente secundaria. Las fronteras semánticas que dividen cada grupo son fluctuantes e inseguras, aunque deben existir áreas centrales bien definidas en cada clase semántica de adjetivos. Por lo demás, hay que contar con los cambios producidos a lo largo de la historia del griego; así, es probable, por ejemplo, que ó́yplos sea en principio un determinativo (como ópєıvós, $\pi \varepsilon \delta\llcorner v o ́ \varsigma$ ), y sólo después haya pasado a calificativo ${ }^{15}$.

Por ello, la clasificación de las clases de adjetivos debe ser resultado del examen de las propiedades morfosintácticas. Así, de los adjetivos

14 Se comprende por ello que muchos adjetivos hayan sufrido variaciones en el curso de la historia del griego. Así, los siguientes adjetivos determinativos crean en época más o menos tardía un comparativo y/o superlativo y un adverbio derivado, circunstancia que demuestra que han pasado a ser calificativos: $\theta \hat{\eta} \lambda u s$.

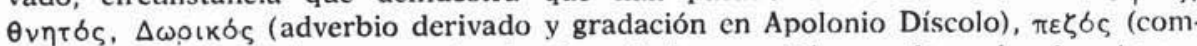

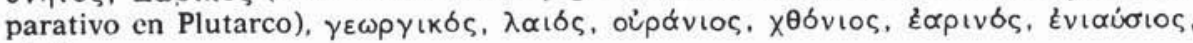
etcétera.

15 Algunos otros ejemplos de uso determinativo de calificativos y viceversa son comentados por L. Bergson, op. cit., p. 54 ss. Entre los determinativos que han



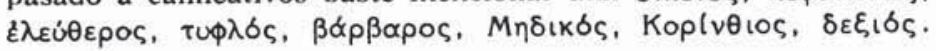


recogidos en la lista precedente bajo el epígrafe de "determinativos» no se documenta en griego, salvo error, ni el comparativo o superlativo ni el adverbio derivado correspondiente. Sí es frecuente, por el contrario, la gradación y la formación de un adverbio derivado en los adjetivos calificativos. En algunos adjetivos, sólo en época tardía $-\mathrm{y}$ en todo caso en fase posterior al estadio sincrónico de los siglos V-IV a. C.se crea el adverbio derivado o se documenta la gradación ${ }^{16}$. Conviene llamar la atención también sobre el hecho de que en ciertos adjetivos la creación del adverbio derivado o del comparativo y superlativo es producto de desarrollos específicos y ampliaciones en el significado de un adjetivo ${ }^{17}$.

En definitiva, las limitaciones de la clasificación semántica afectan sobre todo a la inseguridad sobre la inclusión de un adjetivo en uno u otro grupo. Estas limitaciones pueden ser subsanadas mediante la descripción de las propiedades morfosintácticas, que permiten observar el comportamiento de los ejemplos dudosos en uno u otro sentido. En todo caso, existe cierta correspondencia entre la clasificación semántica y la morfosintáctica.

§ 4. Clasificación según criterios morfológicos. Desde el punto de vista de la descripción morfológica, los adjetivos se caracterizan por poseer marcas que expresan las categorías de la flexión (género, número y caso), y por ser susceptibles de gradación y de formar adverbios derivados ${ }^{18}$.

En realidad, los adjetivos determinativos (como los que integran la lista de ejemplos de § 3) no aparecen en comparativo ni en superlativo, ni tienen adverbio derivado ${ }^{19}$. Lo mismo sucede en los identificadores, con excepción de los demostrativos. Sólo tienen gradación

16 En algunos ejemplos es posible que la ausencia de testimonios se deba a

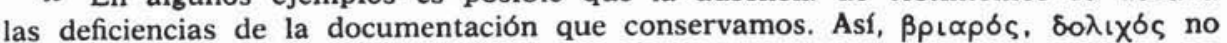
poseen adverbios derivados ni gradación, aunque sí $\mu \dot{\varepsilon} \gamma \alpha \varsigma$ y $\mu \alpha \mathrm{k} \rho \delta \varsigma$, circunstancia que hace insegura su inclusión en uno u otro grupo; lo mismo sucede en ápkios

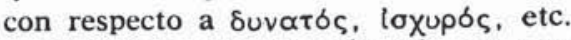

17 Así sucede, por ejemplo, en $\pi \varepsilon \zeta \delta \varsigma, \theta \varepsilon เ \delta \tau \varepsilon \rho \circ \varsigma$. $\delta \varepsilon \xi ı \delta \varsigma, ~ \& v \theta p \omega \pi \varepsilon เ o \varsigma$.

18 Los adverbios derivados de adjetivos no son formas flexivas del adjetivo, cf. H. Pinkster, On Latin Adverbs, Amsterdam-Londres 1972.

19 Algunos ejemplos de cambios diacrónicos ya han sido mencionados en las notas 14 y 15. La lista de ejemplos precedente que ilustra las distintas clases de adjetivos ha sido elaborada del siguiente modo: hemos extraído una lista de los ejemplos mencionados en P. Chantraine, La formation des noms en grec, París 1933, y hemos consultado sus usos en el léxico de LSJ, comprobando qué adjetivos no poseen gradación y/o adverbio derivado, y cuáles poseen ambos, así como la época en la que comienzan a documentarse.

LI, $2.0-9$ 
y son susceptibles de formar adverbios derivados los calificativos, los cuantificadores y los demostrativos.

85. Clasificación según criterios sintácticos. También la descripción sintáctica permite establecer diferencias evidentes entre las distintas clases de adjetivos propuestas.

5.1. El adverbio se define generalmente como modificador opcional de un verbo, un adjetivo $u$ otro adverbio. Sin entrar en la discusión acerca de la corrección de esta definición sintáctica tradicional del adverbio ${ }^{20}$, baste señalar para nuestro propósito que los adjetivos determinativos e identificadores no son susceptibles de ser modificados por un adverbio. Sólo los calificativos y los cuantificadores admiten un adverbio como ampliación opcional:

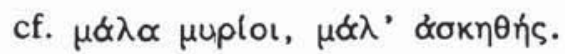

5.2. En la medida en que el adjetivo es ampliación opcional o atributo del nombre, cumple la misma función que el genitivo pertinentivo. Sin embargo, la relación entre genitivo pertinentivo y adjetivo no afecta a todos los adjetivos, sıno sólo a los determinativos y, dentro de los identificadores, a los posesivos. Sólo los determinativos y los posesivos equivalen a nenitivo posesivor.

5.3. También en la rección existen diferencias. Un genitivo sólo puede depender de los cuantificadores, de los calificativos 22 de los interrogativos.

5.4. También existen diferencias, al menos en ático, en el orden de palabras. Según L. Bergson ${ }^{23}$, los determinativos, en general, siguen al nombre, mientras que los calificativos y cuantificadores suelen preceder al nombre.

20 Cf. H. Pinkster, On Latin Adverbs, Amsterdam-Londres 1972, para una discusión detallada.

21 Cf. Schwyzer-Debrunner, p. 176 s. Por supuesto, hacemos aquí abstracción de las diferencias estilísticas entre sintagmas como $v \hat{\eta}^{\prime}$ ' A $\gamma \alpha \mu \varepsilon \mu \nu$ ovénv y $v \hat{\eta}$ ' 'A $\boldsymbol{\alpha} \mu \hat{\varepsilon} \mu$ vovos.

22 El tipo sî $\alpha$ $\theta \alpha \omega \omega v$, Il. XVIII 388, etc., resulta de usar como calificativo el

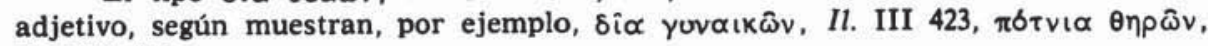
Il. XXI 470.

23 Op. cit., p. 167 s. La misma distinción en el orden de palabras establecen para el latín Marouzeau, op. cit., I, p. 16, y J. N. Adams, IF 81, 1976, p. 88 ss. 
Es cierto, no obstante, que las estadísticas del propio L. Bergson (p. 157 ss.) muestran excepciones a ambas reglas, y quizá sea necesaria una nueva investigación de conjunto sobre este punto ${ }^{24}$. En cuanto a la clase de adjetivos que hemos denominado «identificadores», un recuento parcial de los posesivos e interrogativos en Lisias muestra que $10 \mathrm{~s}$ identificadores suelen preceder al nombre ${ }^{25}$.

5.5. Los adjetivos pueden ser transformados en sustantivos para ejercer las funciones sintácticas propias de los nombres. No obstante, no todos los adjetivos pueden ser sustantivados en las mismas condiciones: los adjetivos calificativos sólo pueden ser sustantivados en el singular no neutro cuando el singular expresa un uso genérico o colectivo ( $\delta \kappa \alpha-$ kós, etc.). Esta restricción no se observa en las restantes clases de adjetivos (cf. Schwyzer-Debrunner, p. 174 s.).

5.6. Una particularidad sintáctica de $\pi \circ \lambda$ ús se refiere al modo de coordinación con otro adjetivo. Cuando uno de los dos o más adjetivos que acompañan a un nombre es $\pi \circ \lambda u ́ s$, el uso habitual es la coordina-

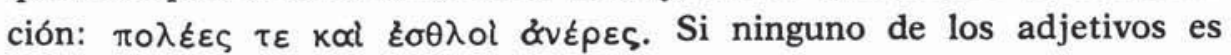
$\pi \circ \lambda u ́ \varsigma$, éstos pueden estar bien coordinados, bien yuxtapuestos: $\kappa \propto \lambda \delta \zeta \varsigma$

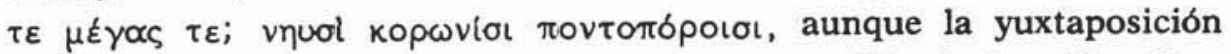
es lo más frecuente (cf. Schwyzer-Debrunner, p. 180 s.). Los identificadores, por su parte, no suelen admitir la coordinación con otros adjetivos (excepto los posesivos).

5.7. Con esta particularidad de $\pi 0 \lambda u ́ s$ se puede poner en relación un uso específico de ciertos adjetivos (cf. Schwyzer-Debrunner, p. 179 s.): un adjetivo que modifica a otro adjetivo en lugar del adverbio corres-

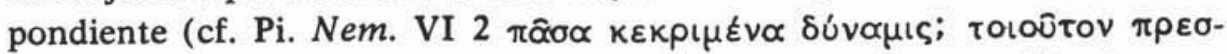

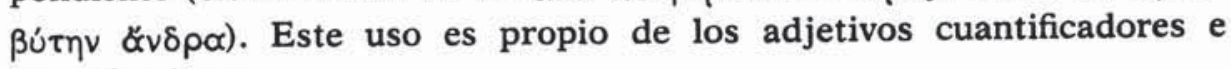
identificadores.

\footnotetext{
24 Un examen somero de algunos adjetivos en Lisias muestra los siguientes datos: $\pi \alpha \hat{\varsigma} \varsigma 119 / 14$ (= 119 veces ante el nombre, 14 tras él), $\pi 0 \lambda u ́ s ~ 104 / 18$, ik $\alpha v \delta \varsigma$

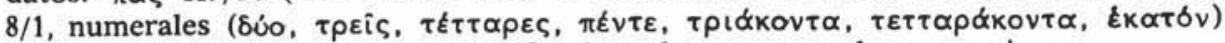
$88 / 17$, t $\delta$ tos $18 / 4$ (es también significativo observar que las excepciones aparecen

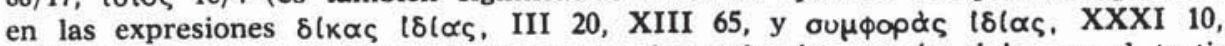
XIII 95, donde el adjetivo es claramente determinativo, según deja ver el testi-

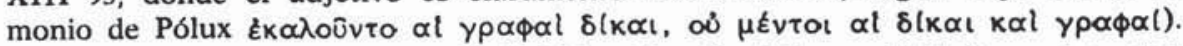

25 Para los demostrativos, cf. J. Palm, Zur Funktion und Stellung des attributiven Demonstrativums im Griechischen, Lund 1960.
} 
5.8. A título de hipótesis quizá se puede añadir una última característica. En los sintagmas en los que aparecen dos adjetivos no acompañados por un nombre, uno de los dos adjetivos ha de estar necesa-

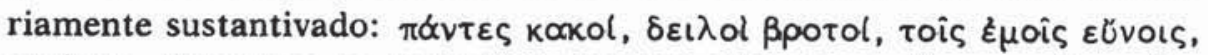
etcétera. Este tipo de sintagmas permite observar cuáles son los adjetivos que son sustantivados con mayor frecuencia, y cuáles son los que admiten más raramente la sustantivación. Por tanto, este criterio para clasificar los adjetivos, si tiene alguna validez, se refiere sólo a la frecuencia de la sustantivación, no a la posibilidad de estar sustantivado. Quizá los adjetivos más raramente sustantivados sean los posesivos, cuantificadores y calificativos ${ }^{26}$.

\$ 6. Clases centrales y clases marginales de adjetivos. A la luz de los resultados precedentes se puede quizá comprender mejor la causa de que la definición semántica tradicional del adjetivo en cuanto clase unitaria de palabras haya invocado la noción de cualidad y haya prestado atención sobre todo a los adjetivos calificativos. La enumeración anterior de las propiedades morfosintácticas de cada clase de adjetivos permite observar que ciertas clases (determinativos e identificadores) manifiestan varias afinidades con la categoría verbal del nombre (cf. $5.1 ; 5.2 ; 5.3 ; 5.4$ ); por el contrario, otras clases de adjetivos (cuantificadores y calificativos) carecen de tales afinidades y, además, expresan ciertas categorías gramaticales (gradación) ajenas al nombre o son susceptibles de formar adverbios derivados (cf. 4). Es natural, pues, que sean estas últimas las clases centrales de adjetivos. Se comprende, por tanto, que la definición semántica tradicional del adjetivo asigne al adjetivo la expresión de la cualidad ${ }^{n}$.

8 7. Conclusión. A modo de conclusión, podemos presentar en el siguiente cuadro las propiedades morfológicas y sintácticas de las distintas clases semánticas de adjetivos en griego antiguo:

26 A diferencia de las lenguas modernas, donde los predicativos verbales suelen ser adjetivos calificativos, en griego cualquier adjetivo puede ser predicativo verbal,

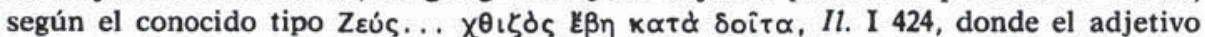

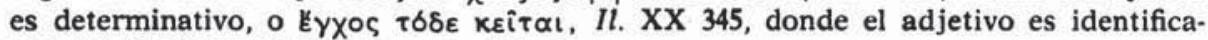
dor (cf. Schwyzer-Debrunner, p. 178 s.).

$n$ Cf. J. Perrot, Langages $50=$ Linguistique et latin, ed. Ch. Touratier, 1978, pp. $17-26$ (esp. p. 20 s.). 
CLASIFICACION DE LOS ADJETIVOS EN GRIEGO

\begin{tabular}{|c|c|c|c|c|c|}
\hline & & \multicolumn{4}{|c|}{ CLASIFICACION SEMANTICA } \\
\hline & & $\begin{array}{c}\text { Determi- } \\
\text { nativos } \\
\end{array}$ & \begin{tabular}{|c|}
$\begin{array}{c}\text { Califica- } \\
\text { tivos }\end{array}$ \\
\end{tabular} & $\begin{array}{l}\text { Cuantifi- } \\
\text { cadores }\end{array}$ & $\begin{array}{l}\text { Identifi- } \\
\text { cadores }\end{array}$ \\
\hline \multirow{2}{*}{ 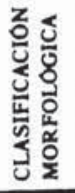 } & Posibilidad de gradación, cf. 4. & - & + & + & 一 \\
\hline & Formación de adverbios derivados, cf. 4. & - & + & + & $\overline{(+)}$ \\
\hline \multirow{8}{*}{ 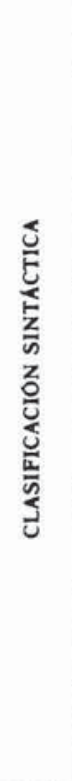 } & Modificación mediante un adverbio, cf. 5.1. & - & + & + & - \\
\hline & $\begin{array}{l}\text { Equivalencia funcional con genitivo } \\
\text { posesivo, cf. } 5.2 \text {. }\end{array}$ & + & - & 一 & $\overline{(+)^{* * *}}$ \\
\hline & Posibilidad de regir un genitivo, cf. 5.3. & - & + & + & $(\overline{+}) * \cdots$ \\
\hline & Orden de palabras ante el nombre, cf. 5.4. & - & + & + & + \\
\hline & Restricciones en la sustantivación, cf. 5.5. & - & + & - & - \\
\hline & Coordinación de dos adjetivos, cf. 5.6. & $+1-$ & $+1-$ & + & - \\
\hline & $\begin{array}{l}\text { Adjetivo (en lugar de adverbio) modificador } \\
\text { de adjetivo, cf. } 5.7 \text {. }\end{array}$ & - & 一 & + & + \\
\hline & $\begin{array}{l}\text { Sustantivación en sintagmas de dos } \\
\text { adjetivos, cf. 5.8. }\end{array}$ & + & - & - & $\stackrel{+}{(-) * *}$ \\
\hline
\end{tabular}

"Adjetivos demostrativos; "* adjetivos posesivos; "** adjetivos interrogativos

\section{Emilio Crespo}

